\title{
Hexokinase Isozyme Variability in Drosophila robusta
}

\author{
Conrad Knutsen, ${ }^{1}$ Charles F. Sing, ${ }^{1}$ and George J. Brewer ${ }^{1,2}$ \\ Received 7 April 1969-Final 8 June 1969
}

Several isozymes of hexokinase have been found in Drosophila robusta. These have been defined in this paper in several dimensions, including genetic variation, ontogenic variation, tissue-organ variation, and substrate specificity.

\section{INTRODUCTION}

Electrophoretic studies of Drosophila have demonstrated numerous examples of genetically determined polymorphism, ontogenetic variation, and tissue-organ variation (Beckman and Johnson, 1964; Johnson et al., 1968). The present work defines the hexokinase isozymes in Drosophila robusta in all of these dimensions, with additional studies of substrate specificity.

\section{MATERIALS AND METHODS}

Starch gel electrophoresis was performed according to the method developed for the study of human red cell hexokinase isozymes by Brewer and Knutsen (1968). The gel buffer was $0.021 \mathrm{M}$ tris, $0.021 \mathrm{M}$ boric acid, and $0.001 \mathrm{M}$ EDTA (ethylenediaminetetraacetic acid) adjusted to $p \mathrm{H} 8.4$ with $1 \mathrm{~N}$ hydrochloric acid. The electrode buffer consisted of $0.21 \mathrm{M}$ tris, $0.15 \mathrm{M}$ boric acid, and $4.7 \times 10^{-3} \mathrm{M}$ EDTA adjusted to $\mathrm{pH} 8.0$

The work reported in this paper was supported in part by USPHS grant AM 09381, USPHS Career Development Award 1-K3-AM 7959 (GJB), and by Contract AT (11-1)-1152, Atomic Energy Commission, and in part by the Research and Development Command, Office of the Surgeon General, Department of the Army, under Contract DA-49-193-MD-2855 with the Department of Medicine, University of Michigan. This is contribution No. 662 from the Army Research Program on Malaria.

${ }^{1}$ Department of Human Genetics, University of Michigan, Ann Arbor, Michigan.

2 Department of Medicine (Simpson Memorial Institute), University of Michigan, Ann Arbor, Michigan. 
with concentrated hydrochloric acid. Electrophoresis was carried out for $18-20 \mathrm{hr}$ at a potential of $12.5 \mathrm{v} / \mathrm{cm}$ at $4 \mathrm{C}$.

The gels were developed for $2.5-3 \mathrm{hr}$ at $37 \mathrm{C}$ in $0.05 \mathrm{M}$ tris buffer, $p \mathrm{H} 8.0$, containing $1 \times 10^{-2} \mathrm{M}$ magnesium chloride, $2.4 \times 10^{-3} \mathrm{M}$ adenosine triphosphate (ATP), $1.2 \times 10^{-1} \mathrm{M}$ nicotinamide adenine dinucleotide phosphate (NADP), 7 international units $(0.05 \mathrm{mg})$ of glucose 6-phosphate dehydrogenase (Boehringer), $2.4 \times 10^{-4} \mathrm{M}$ 3-(4, 5-dimethylthiasolyl-2) 2,5-diphenyltetrazolium bromide (MTT tetrazolium), and $2.4 \times 10^{-4} \mathrm{M}$ phenazine methosulfate. When staining for glucokinase, $8.3 \times 10^{-2} \mathrm{M}$ glucose was included in the developing buffer. When staining for fructokinase, $8.3 \times 10^{-3} \mathrm{M}$ fructose was substituted for the glucose and 8.5 international units $(0.11$ $\mathrm{mg}$ ) of phosphohexose isomerase (Sigma) was added to complete the staining reaction. Laboratory stocks of Drosophila robusta were obtained from Dr. H. Carson of Washington University. Flies selected at random from each of these stocks served as the source to generate a larger population on which these studies were performed. Lines homozygous for hexokinase alleles at two loci were established to provide material to test the genetic basis of the variability present at these loci.

Individual flies were ground in $0.02 \mathrm{ml}$ of the gel buffer. Larvae or pupae were also individually ground in a similar manner. To study tissue and organ distribution of the isozymes, dissections were performed in physiological saline after dipping the flies in $95 \%$ ethanol to facilitate wetting. The various tissues were each ground on $0.02 \mathrm{ml}$ of the starch gel buffer. The extracts were then applied to slots in the starch gels.

\section{RESULTS}

\section{Nomenclature}

Starch gel zymograms of adult flies are shown in Fig. 1. The gel patterns are similar to those of Murray, who used a different starch gel system for their studies of the hexokinase isozymes of D. melanogaster (Murray and Ball, 1967).

Murray has divided the gel pattern in his system into five zones labeled A through $\mathrm{E}$ beginning with the most cathodal zone. This order is the reverse of the usually accepted method, which consists of labeling the most anodal zone A, or 1. For this reason, we have utilized an alternative method. The gel is divided into four regions of hexokinase activity, each consisting of a number of bands. Bands 1 and 2 are in region 1 , bands $3-8$ in region 2 , bands $9-12$ in region 3 , and band 13 is in region 4 (Fig. 1). The bands have been grouped in this manner to correspond with genetic, ontogenetic, tissue origin, and substrate specificity differences.

The tentative phenotypic nomenclature for this system rests on our present assumption that there are at least four loci controlling the production of the hexokinases. Each locus controls the production of the hexokinase of each region. We can number them 1 to 4 , with each locus having the same number as the respective regions. Hexokinase 3 ( Hex 3 ) is then the locus controlling the hexokinases of region 3. If variation has been found in a particular region, the alleles are each given a letter designation. 


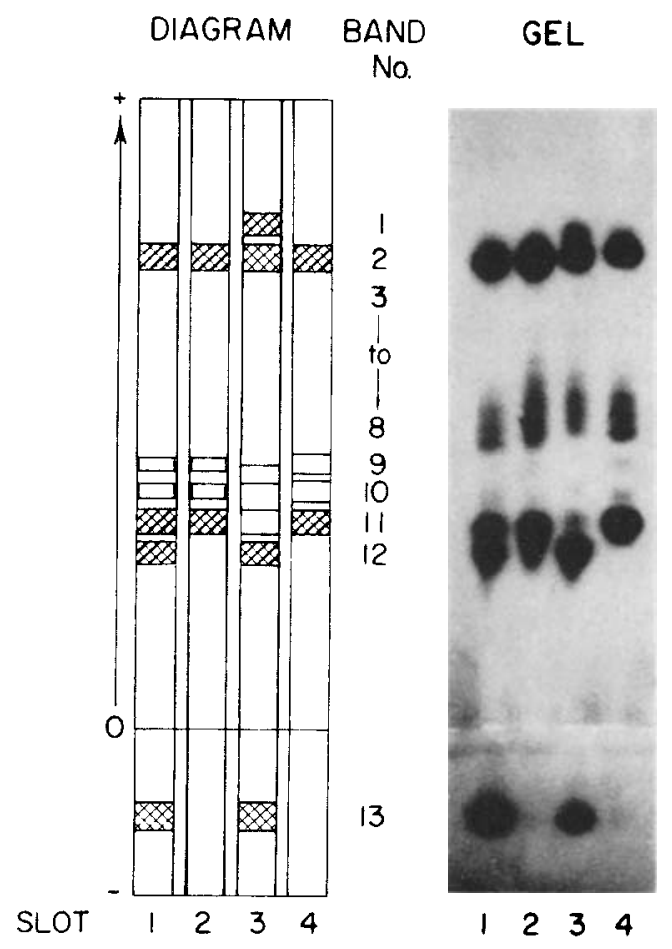

Fig. 1. Starch gel hexokinase isozyme patterns of whole fly extracts of Drosophila robusta. Gel pattern is divided into four regions, region 1 consisting of bands 1 and 2, region 2 consisting of bands 3-8, region 3 consisting of bands 9-12, and region 4 consisting of band 13. Extracts in slots 1 and 3 are from adult males and those from slots 2 and 4 are from adult females. Genetic variation discovered in region 3 (bands 9-12) is also shown. The faster-migrating band (band 11 , slots 2 and 4 ) is the result of a double dose of the $A$ allele of the gene controlling hexokinase-3. The slower-migrating band (band 12, slot 3) is due to the $B$ allele of the same gene. The heterozygous form is pictured in slot 1 , bands 11 and 12 . Variation in migration of minor bands ( 9 and 10) in unison with bands 11 and 12 can be seen by comparing slots 3 and 4 .

\section{Genetic Studies}

\section{Region 3 (Bands 9-12)}

Region 3 shows the least genetic complexity. The mode of inheritance for region 3 is clear; it is a simple, codominant, two-allele system (Table I). The phenotype showing a single faster-migrating band (band 11, slots 2 and 4, Fig. 1) is the result of the presence of two $A$ alleles of Hex 3 locus. A single slower-migrating band (band 12, 
Table I. Mating Data from Region 3 Isozyme Phenotypes

\begin{tabular}{lrrr}
\hline & \multicolumn{3}{c}{ Offspring phenotypes } \\
\cline { 2 - 4 } Mating types & A/A & A/B & B/B \\
\hline A/A $\times$ A/A & 48 & 0 & 0 \\
$\mathrm{~B} / \mathrm{B} \times \mathrm{B} / \mathrm{B}$ & 0 & 0 & 30 \\
$\mathrm{~A} / \mathrm{A} \times \mathrm{B} / \mathrm{B}$ & 0 & 36 & 0 \\
$\mathrm{~A} / \mathrm{B} \times \mathrm{A} / \mathrm{B}$ & 22 & 56 & 17 \\
$\mathrm{~A} / \mathrm{A} \times \mathrm{A} / \mathrm{B}$ & 19 & 13 & 0 \\
\hline
\end{tabular}

\section{A} DIAGRAM BAND

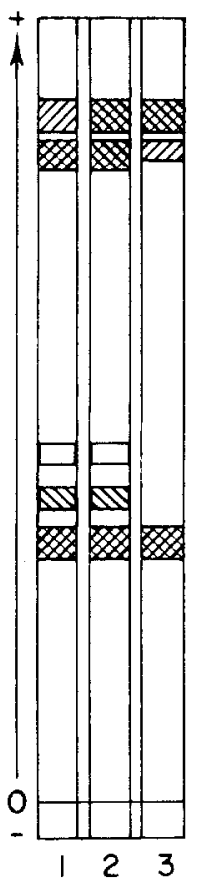

No.

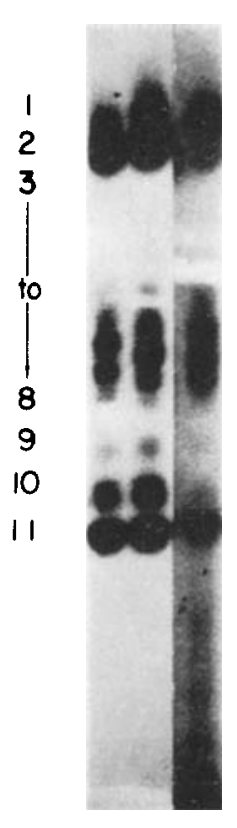

123
B GEL

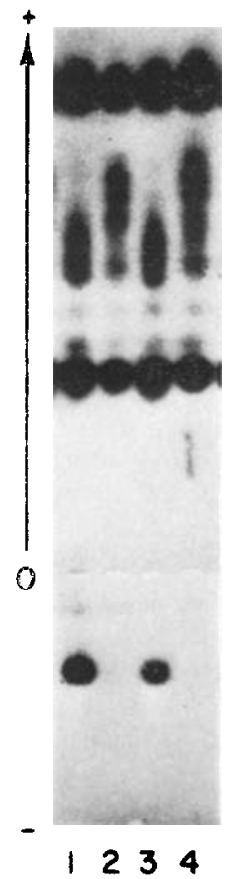

Fig. 2. (a) Starch gel hexokinase isozyme patterns of whole fly extracts of Drosophila robusta. Genetic variation found in region 1 is illustrated. Dose of two $B$ alleles results in production of hexokinase-1 (band 2, slot 1). Dose of two faster alleles results in production of hexokinase-1 (band 1, slot 3). Presence of single dose of both alleles results in the heterozygous form (bands 1 and 2, slot 2). Band 2 in slot 1 and band 1 in slot 3 are muscle-derived isozymes, while band 1 in slot 1 and band 2 in slot 3 are part of the isozyme pattern originating from the digestive tract. The phenomenon of two isozymes overlying each other has caused difficulty in scoring between a homozygote $B$ and the true heterozygote. (b) Variation in region 2 is shown. Slots 1 and 3 are male adult fly extracts, and 2 and 4 are female adult fly extracts. Bands in region 2 in slots 2 and 4 migrate slightly faster than those in slots 1 and 3 . 
slot 3, Fig. 1) is the result of the presence of both $B$ alleles of Hex 3. The heterozygous $A B$ results in the appearance of a double band (bands 11 and 12, slot 1, Fig. 1). A pair of minor bands (bands 9 and 10, slots 1, 2, and 4, Fig. 1) show differences in migration in unison with the genetic variation in the major bands (illustrated in slot 3, Fig. 1). Sex linkage with this region has been excluded upon observing male-tomale transmission involving each of the two alleles present in the population.

\section{Region 1 (Bands I and 2)}

Region 1 is more complex than region 3 [Fig. 2 (a)]. Isozymes from two tissues (digestive and muscle see Tissue Distribution section of this paper) migrate to identical positions in this region. Isozymes from digestive tissues form bands in the 1 and 2 position and show no apparent genetic variation. Isozymes from muscle, on the other hand, do show genetic variation. The tentative explanation for variation seen in this region is similar to that for region 3; that is, the mode of inheritance appears to be due to a simple, codominant, two-allele system. The phenotype showing a single faster-migrating band [band 1, slot 3, Fig. 2(a)] is the result of the presence of two $A$ alleles of the locus Hex 1. A single slower-migrating band is due to the presence of two $B$ alleles of Hex $I$ [band 2, slot 1, Fig. 2(a)]. In the heterozygous form the double band is the result of the presence of both the $A$ and $B$ alleles of Hex $I$ [bands 1 and 2, slot 2, Fig. 2(a).]. However, results from breeding experiments designed to evaluate the genetics of this system have been confounded by what appears to be a greatly increased mortality rate in the presence of a double dose of the $A$ allele. The increased mortality rate appears to be related to a severe wing defect, which was exhibited by both of the flies which were found to have only the faster band [band 1, slot 3, Fig. 2(a)]. Our genetic analysis of region 1 is tentative. In addition to the increased mortality rate, the fact that isozymes from the muscle and digestive tissues migrated to the same area has added to the difficulty in scoring for purposes of analyzing the results of mating data. This difficulty can be overcome by tissue dissection, but this is tedious. More recently we have utilized substrate differences in the isozymes, which should facilitate analysis of results in the future (see Substrate Specificity section).

\section{Region 2 (Bands 3-8)}

Region 2 consists of a series of about six lighter-staining isozymes. During electrophoresis of the several hundred flies used in this study, some variation in the rate of migration was observed in region 2 . The variation involves shifting of the whole series of isozymes [Fig. 2(b)]. Slots 1 and 3 show relatively slower migration. While we have noticed some association with sex (females tend to exhibit patterns with faster migration), the genetics of this region has not as yet been elucidated. The apparent association with sex has confounded the forming of any hypothesis concerning the mode of inheritance using our present data. 


\section{Region 4}

The cathodally migrating band of region 4 shows complete association with the male sex [band 13, slots 1 and 3, Fig. 1 and 2(b); slot 2, Fig. 3 (a, b)]. This hexokinase isozyme occurs in the testes, as reported by Murray and Ball (1967) for D. melanogaster.

\section{Tissue Distribution}

The tissues we have studied include the testes, ovaries, digestive tissues, and muscle

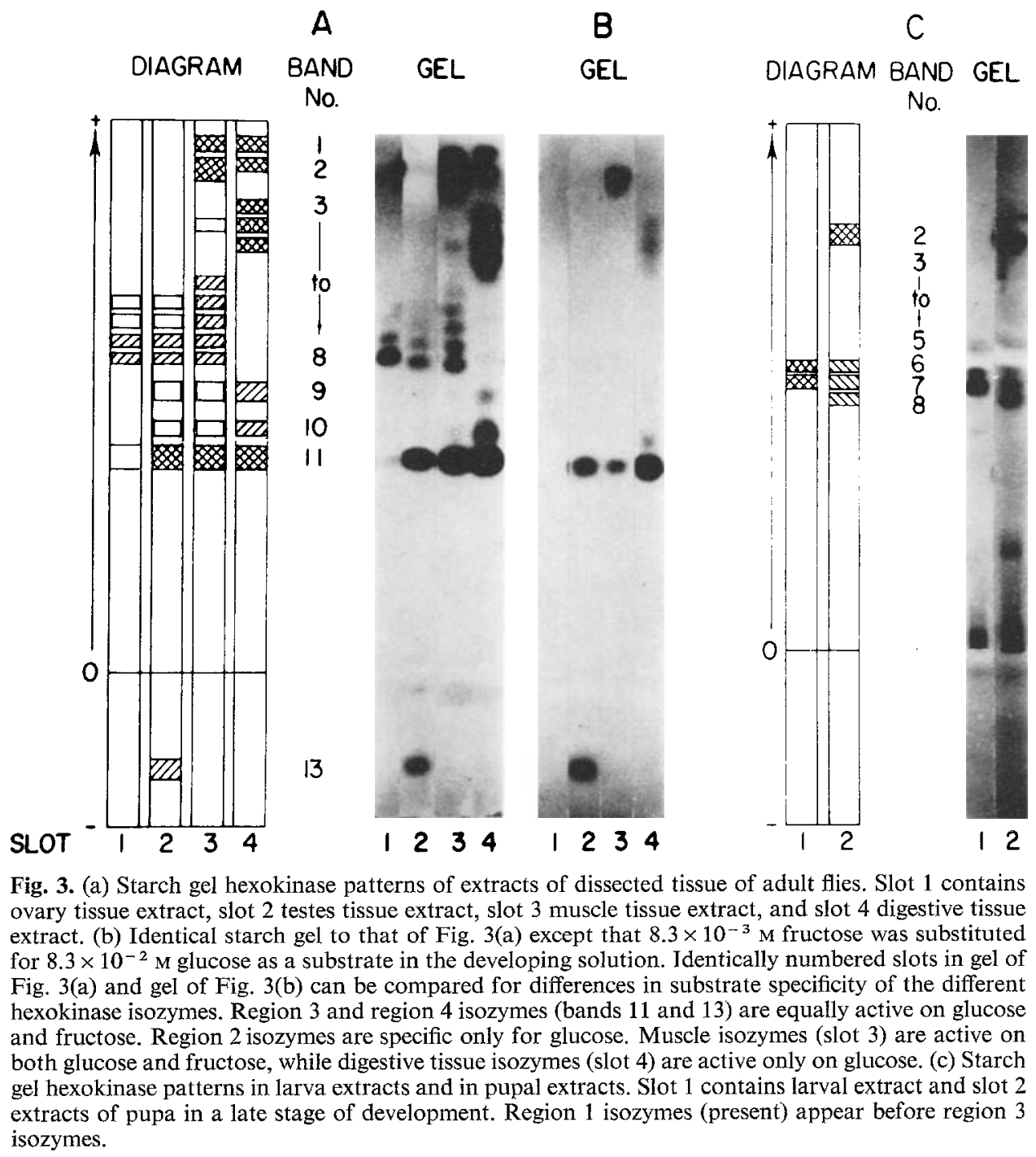


tissue from the ventral thorax and legs. The distribution of isozymes in the various tissues is shown in Fig. 3(a).

The hexokinases of region 2 , with some variation in quantitative activity and in number of bands, occur in all of the tissues studied. Extracts of testes, ovaries, and muscle exhibited the slower-migrating isozymes of this region, while in the digestive system extracts, faster-migrating isozymes were evident [Fig 3(a)]. In whole fly extracts these faster-migrating isozymes appeared rather faint, probably because of dilution.

Region 1 isozymes originate from two different tissues-muscle, which stains very intensely, and digestive tissues. The muscle isozyme shows genetic variation in region 1. As noted previously, we have had some difficulty in analyzing genetic variation in bands from the muscle tissue because two bands from the digestive tissues, which show no variation, migrate to approximately the same place.

Hexokinase of region 3 appears in extracts of the testes, muscle, and the digestive system [slots 2-4, Fig. 3(a)], but only weakly if at all in the ovary tissues [slot 1, Fig. 3(a)]. Region 4 consists of the testis-specific band [slot 2, Fig. 3(a)].

\section{Ontogenetic Studies}

Drosophila in various stages of development were also examined with respect to the hexokinase isozymes.

In eggs, no hexokinase activity was observed using up to six eggs per gel slot. In larvae, the hexokinases of region 2 were evident throughout the instar stages [slot 1 , Fig. 3(c)]. In the pupal stage the hexokinases of regions 1 and 3 become evident, and their appearance seems to occur in orderly fashion, coinciding with the development of the adult forms within the pupal case [slot 2, Fig. 3(c)]; that is, region 1 appears before region 3 .

\section{Substrate Specificity}

After electrophoresis the starch gels were sliced horizontally into two slices. In the solution used to develop one of the gel slices, fructose was substituted for glucose [compare slots 1-4 of Fig. 3(a) with 1-4 of Fig. 3(b)]. The hexokinases of regions 3 and 4 were equally as active on fructose and glucose. Isozymes in region 1 were considerably less active with fructose than with glucose. Experiments involving tissue dissection showed that only the isozymes of region 1 originating from the muscle tissue were active with fructose [slot 3 of Fig. 3(b)], while the region 1 isozymes originating from the digestive tract were not [slot 4, Fig. 3(b)].

\section{Other Species}

The hexokinase system was examined in other species as well as D. robusta. Approximately 35 adult individuals of $D$. melanogaster and 5 adult individuals each of $D$. prosaltans and $D$. willistoni were studied in our system. Except for differences in the rate of migration of the isozymes between species, the hexokinase patterns of these 
three species are very similar to that found in D. robusta. Variation has been found in region 1 and region 3 hexokinases in $D$. melanogaster. One difference present was the direction of migration of the testes-specific band in D. melanogaster. The testesspecific band in $D$. melanogaster migrated anodally instead of cathodally as in $D$. robusta.

\section{DISCUSSION}

The number of hexokinase isozymes found in D. robusta is most impressive. To date, this degree of multiplicity in Drosophila has been surpassed only by the number of esterase isozymes, which are, of course, revealed by a nonspecific stain and may include several unrelated groups of enzymes. The proportion of hexokinase loci segregating (two of at least four loci) is considerable, but in agreement with predictions from earlier estimates on the proportion of loci which are polymorphic in natural populations (Shaw, 1965; Lewontin and Hubby, 1966; Harris, 1966).

Differences have been found in relation to substrate specificity. All of the isozymes were active with glucose, while only a few were active with fructose. Possibly fructose and other sugars normally ingested by the fly are ordinarily converted to glucose for utilization.

Our observations on this system suggest the following speculations:

1. The number of isozymes found in a tissue may be related to its degree of functional specialization. The reproductive organs, which carry out a similar function under all conditions, produce a lesser number of isozymes than do the gut and muscle, which in the adult must be more flexible and function in a variety of ways under varying conditions. Also, tissues such as the digestive system and skeletal muscle system have a number of subdivisions or areas of functional specialization. It may be that the isozymes found in gut and muscle tissues are distributed throughout the divisions of these systems, each division possibly having slightly different internal conditions. As previously suggested by others, the organism may have developed isozymes particularly suited to its environmental conditions. Such a hypothesis predicts that the number of isozymes in an organism will increase as the complexity of the organism increases. That this may be the case is supported by data reported by Brewer and Sing (1968). Evolution of an isozyme in the particular environmental conditions of an organ or tissue may be an efficient way by which a multicellular organism can deal with its complexity.

2. Larval forms show fewer hexokinase isozymes than adult forms. Specific isozyme bands derived from the digestive and muscle tissues of the adult are not found in the comparable tissues of the larva. Larval tissues go through a process of histolysis during pupation and are replaced by their adult forms. The additional isozymes in the digestive and muscle tissues of the adult may be an adaptive response to increased internal variability and external environmental variability associated with the emergence of the adult organism.

3. Multiple molecular forms of enzymes may be partitioned into two types, those that are due to genetic segregation and those that are constant in the members of the population (nonsegregating). Segregating hexokinase isozymes tend to be more 
prevalent in specialized tissues of the adult. The mixture of segregating and nonsegregating isozymes as illustrated by the hexokinases of Drosophila deserves comment. First, most of the hexokinase isozymes are nonsegregating. Second, nonsegrating isozymes tend to be found throughout most of the life cycle, while those present during only part of the life cycle, such as those derived from muscle, exhibit genetic polymorphism. Investigations are under way to determine that a widespread development of nonsegregating isozymes occurs in response to variation in the internal environment of the individual, while polymorphism exists at some of the loci expressed in the adult which may enable the population to adapt to external environmental variation.

\section{REFERENCES}

Beckman, L., and Johnson, F. M. (1964). Esterase variation in Drosophila melanogaster. Hereditas 51: 212 .

Brewer, G. J., and Knutsen, C. A. (1968). Hexokinase isozymes in human erythrocytes. Science 159: 650 .

Brewer, G. J., and Sing, C. F. (1968). The study of evolution through isozymes. J. Clin. Invest. 46: 11a (abst.).

Harris, H. (1966). Enzyme polymorphisms in man. Proc. Roy. Soc. (London) B164: 298.

Johnson, F. M., Richardson, R. H., and Kambysellis, M. P. (1968). Isozyme variability in species of the genus Drosophila. IV. Distribution of the esterases in the body tissues of D. aldrichi and D. mulleri adults. Biochem. Genet. 1: 249.

Lewontin, R. C., and Hubby, J. L. (1966). A molecular approach to the study of genic heterozygosity in natural populations. II. Amount of variation and degree of heterozygosity in natural populations of Drosophila pseudoobscura. Genetics 54: 595.

Murray, R. F., and Ball, J. A. (1967). Testis-specific and sex-associated hexokinases in Drosophila melanogaster. Science 156: 81.

Shaw, C. R. (1965). Electrophoretic variation in enzymes. Science 149: 936. 